\title{
Evaluation of Some Pigeonpea Genotypes for Their Response against Pod Fly, Melanagromyza obtusa (Malloch) Infestation
}

\author{
Badabagni Chiranjeevi $^{1^{*}}$, Neetin R. Patange ${ }^{2}$ and Prashant G. Chandile ${ }^{3}$ \\ ${ }^{1}$ Madhavi Farms, Sakalavara Village, Bangalore-560083, India \\ ${ }^{2}$ National Agricultural Research Project, Aurangabad-431005 \\ (VNMKV, Parbhani), M.S., India \\ ${ }^{3}$ College of Agriculture, Naigaon (B)-431709 (VNMKV, Parbhani), Dist. Nanded (M.S.), India \\ *Corresponding author
}

\section{A B S T R A C T}

A field experiment was conducted at Agricultural Research Station, Badnapur (Vasantrao Naik Marathwada Krishi Vidyapeeth (VNMKV), Parbhani), India during Kharif season of

\section{Keywords}

Infestation,

Maggots,

Melanagromyza

obtusa, Pigeonpea,

Pod fly, Population and Pupae

\section{Article Info}

Accepted:

12 February 2018

Available Online:

10 March 2018
2015-2016. A total of 20 pigeonpea genotypes were screened for their response against Melanagromyza obtusa Malloch infestation. The present study revealed that the first incidence of $M$. obtusa maggots and pupae was observed in the $44^{\text {th }}$ standard meteorological week (39.87 maggots and 10.33 pupae per 100 pods) and attained its peak on $03^{\text {rd }}$ standard meteorological week with 120.78 maggots and 55.05 pupae per 100 pods, thereafter its population declined due to maturity of grains and reached to minimum on $07^{\text {th }}$ standard meteorological week in all the genotypes. The highest population of $M$. obtusa was recorded in BRG-2 (277.64 maggots and 101.26 pupaeper 100 pods) and followed by BRG-1 (206.60 maggots and 81.93 pupae per 100 pods) indicating that the genotypes were highly susceptibile to $M$. obtusa. Wherein, the lowest population of $M$. obtusa was recorded in Cajanus cajanifolius (3.98 maggots and 0.75 pupae per 100 pods) and followed by V-127 (10.35 maggots and 3.38 pupae per 100 pods) indicating their high level of resistance against $M$. obtusa, while no population of $M$. obtusa was observed in Cajanus scarabaeoides indicating that the genotype is having most resistance against $M$. obtusa infestation and it can be used in breeding programmes in order to produce resistant cultivars for the benefit of farmers.

\section{Introduction}

Pigeonpea (Cajanus cajan (L.) Millsp.) is grown throughout the tropics, but most widely in south and southeast Asia. Pigeonpea is one of the most important pulse crop, widely grown by small farmers in the semi-arid tropics as a backyard subsistence crop being cultivated in more than 25 countries of the world. India is largest producer of pigeonpea, contributing more than 90 per cent of the world's production. However, its productivity is far below than potential yield. The major constraint for low yield is heavy infestation of insect pest complex during reproductive phase of the crop. More than 200 species of insects 
feed on this crop, of which the pod fly, Melanagromyza obtusa (Malloch) (Diptera: Agromyzidae), commonly known as pigeonpea pod fly or red-gram pod fly or turpod fly is a major pest; attacking pigeonpea and act as a key pest causing heavy crop losses in India (Lateef and Reed, 1990; Shanower et al., 1999; Kumar and Nath, 2003; Kumar et al., 2003; Nath et al., 2008). It is a monophagous species and devours the developing seeds of pigeonpea crop. The damage caused by $M$. obtusa maggots is much beyond economic level. A single larva in its life time consumes and destroys one complete seed and sometimes it has been seen to move to adjacent seed of the same pod to continue the feeding if the first seed could not fulfill its requirements (Ipe, 1974). Losses due to pod fly damage have been estimated to be US\$ 256 millions annually (Sharma et al., 2011). Many researchers in India have identified different lines such as PDA88-2E, PDA89-2E, IC245312, IC468105, IC489744, IC490149, IC490388, IC525402, ICPL11964, ICP1053, PDA 89-2E, PDA 882-E, PDA 91-1E, PDA 92-2E, PDA 93-2E, ICPL 82064-E15-E1, Phule T25, T32, KP 4769, C-11 and BDN-1 to provide moderate resistance to the pigeon pea pod fly (Singh et al., 2013). Different cultivars have different levels of damage. The potential for developing cultivars with high levels of resistance appears to be good (Shanower et al., 1998). Identification and cultivation of cultivars which are less preferred by pod fly have number of advantages, particularly for an eco-friendly management of pigeonpea pod fly. However, Singh and Singh (1990) reported that no definite conclusions could be drawn about the relative susceptibility of pigeonpea genotypes to pod fly damage because of staggered flowering and variation in pod fly abundance overtime. Since levels of resistance to thispest in the cultivated pigeonpea are low to moderate, it is important to identify pigeonpea cultivar that permits slow growth or lesser population buildup of pod fly. Therefore, keeping the view above the present investion is entitled 'evaluation of some pigeonpea genotypes against pod fly, Melanagromyza obtusa (Malloch) infestation' that will beneficial to farmers as well as useful in breeding programmes to produce the resistant genotypes.

\section{Materials and Methods}

A field experiment was conducted in randomized block design with eleven treatments replicated thrice at Research Farm, Agricultural Entomology Unit, Agricultural Research Station, Badnapur (Vasantrao Naik Marathwada Krishi Vidyapeeth (VNMKV), Parbhani), Maharashtra, India during Kharif season of 2015-2016 to study the response of 20 pigeonpea genotypes against pod fly, Melanagromyza obtusa (Malloch) infestation. The plot size was three rows of $5.4 \mathrm{~m}$ length $\left(5.4 \mathrm{~m} \times 2.4 \mathrm{~m}=12.96 \mathrm{~m}^{2}\right)$ and the row to row and plant to plant distance was maintained at $60 \mathrm{~cm}$ and $30 \mathrm{~cm}$, respectively.

All recommended cultural and agronomical practices were followed homogeneously in all the genotypes to raise a good crop. None of the insecticide was applied to protect the crop from infestation of $M$. obtusa. The genotypes were raised under rainfed conditions and only a protective irrigation was provided during flowering stage of the crop. The pest reaction was recorded from pod initiation till harvest of the crop. Hand picking of pod borer, Helicoverpa armigera (Hubner) larvae and other pest on pods was done to avoid the losses caused by these pests. The population i.e. maggots and pupae of M. obtusa was counted from 100 randomly selected pods covering all the plants of each genotype on weekly basis (Keval et al., 2010; and Chiranjeevi and Patange, 2017). All the data recorded were subjected to statistical analysis as per the randomized block design procedure to draw meaningful conclusions. 


\section{Results and Discussion}

The data on mean maggot population of pod fly, Melanagroyza obtusa (Malloch) on 20 different pigeonpea genotypes under study during $44^{\text {th }}$ standard meteorological week (SMW) to $07^{\text {th }}$ SMW is presented in Table 1. It is clear from the data that all genotypes indicated significant differences with regard to maggot population of pigeonpea pod fly, $M$. obtusa. The maggot population of $M$. obtusa varied from 1.67 to 306.67 maggots per 100 pods during crop season (i.e. $44^{\text {th }}$ to $07^{\text {th }}$ SMW). The first incidence of M. obtusa was observed in the $44^{\text {th }}$ SMW and remained active till $07^{\text {th }} \mathrm{SMW}$ in most of the pigeonpea genotypes under study. The maggot population of $M$. obtusa ranged from 25.67 to 149.69 maggots per 100 pods in BDN-2, 5.67 to 45.00 maggots per 100 pods in BDN-2010$1,14.67$ to 111.67 maggots per 100 pods in BDN-2013-41, 15.00 to 149.67 maggots per 100 pods in BDN-2014-1, 27.67 to 97.67 maggots per 100 pods in BDN-2014-3, 31.00 to 109.67 maggots per 100 pods in BSMR$736,63.67$ to 164.00 maggots per 100 pods in BSMR-846, 14.00 to 95.00 maggots per 100 pods in KALITUR, 46.00 to 166.27 maggots per 100 pods in KHADKI, 14.67 to 116.67 maggots per 100 pods in GULYAL, 99.67 to 261.00 maggots per 100 pods in BRG-1, 214.00 to 306.67 maggots per 100 pods in BRG-2, 36.67 to 148.67 maggots per 100 pods in LRG-41, 112.67 to 248.67 maggots per 100 pods in ICP-7035, 17.67 to 56.50 maggots per 100 pods in ICP-10531, 43.67 to 100.67 maggots per 100 pods in ICP-322, 12.00 to 45.00 maggots per 100 pods in BSR-1, 2.00 to 22.67 maggots per 100 pods in $\mathrm{V}-127$ and 1.67 to 20.67 maggots per 100 pods in Cajanus cajanifolius, respectively, wherein, no $M$. obtusa maggots was observed in Cajanus scarabaeoides, indicating high resistance against $M$. obtusa. The peak maggot population of pod fly irrespective of genotype was in $03^{\text {rd }}$ SMW and thereafter, it declined due to maturity of grains and reached to minimum on $07^{\text {th }}$ SMW in all the genotypes. The maggot population of $M$. obtusa on different pigeonpea genotypes in descending order was $03^{\text {rd }}>02^{\text {nd }}>01^{\text {st }}>04^{\text {th }}>52^{\text {nd }}>51^{\text {st }}>$ $05^{\text {th }}>50^{\text {th }}>49^{\text {th }}>48^{\text {th }}>06^{\text {th }}>47^{\text {th }}>46^{\text {th }}>07^{\text {th }}>$ $45^{\text {th }}>44^{\text {th }}$ SMW during the crop period.

The highest maggot population of M. obtusa (Malloch) was recorded in BRG-2 (277.64 maggots per 100 pods), followed by BRG-1 (206.60 maggots per 100 pods), ICP-7035 (187.02 maggots per 100 pods), BSMR-846 (112.08 maggots per 100 pods), LRG-41 (99.73 maggots per 100 pods), KHADKI (97.74 maggots per 100 pods), BDN-2 (93.23 maggots per 100 pods) and BDN-2014-1 (85.79 maggots per 100 pods), respectively. The lowest mean maggot population of $M$. obtusa (Malloch) was recorded in the genotype, $C$. scarabaeoides (no maggots), $C$. cajanifolius (3.98 maggots per 100 pods), followed by V-127 (10.35 maggots per 100 pods), BDN-2010-1 (22.27 maggots per 100 pods), BSR-1 (28.46 maggots per 100 pods) and ICP-10531 (35.78 maggots per 100 pods), respectively, while, the genotypes, KALITUR (52.02 maggots per 100 pods), BDN-2014-3 (55.33 maggots per 100 pods), BDN-2013-41 (56.92 maggots per 100 pods), GULYAL (69.40 maggots per 100pods), ICPL-322 (73.50 maggots per 100 pods) and BSMR-736 (73.67 maggots per 100 pods), respectively shown intermittent moggot population of $M$. obtusa. The present findings are in accordance with; Sharma et al., (2003), who reported that accessions belonging to Cajanus scarabaeoides (L.) Thouars showed resistance against pod fly. Keval et al., (2010) reported that the first incidence of pod fly was observed in the $4^{\text {th }}$ standard week and remained active till $12^{\text {th }}$ standard week in all the varieties, respectively, wherein, the peak population of pod fly irrespective of variety was in $9^{\text {th }}$ standard week and thereafter, it declined due to maturity of grains. 
Table.1 M. obtusa (Malloch) maggots population in different pigeonpea genotypes

\begin{tabular}{|c|c|c|c|c|c|c|c|c|c|c|c|c|c|c|c|c|c|}
\hline \multirow[t]{2}{*}{ GENOTYPE } & \multicolumn{17}{|c|}{ Maggot Population per 100 Pods per SMW } \\
\hline & 44 & 45 & 46 & 47 & 48 & 49 & 50 & 51 & 52 & 01 & 02 & 03 & 04 & 05 & 06 & 07 & Mean \\
\hline \multirow[t]{2}{*}{ BDN-2 } & 25.67 & 45.00 & 55.00 & 67.67 & 78.00 & 88.67 & 108.67 & 118.67 & 124.67 & 134.00 & 141.00 & 149.67 & 129.00 & 104.00 & 76.00 & 46.00 & 93.23 \\
\hline & $(5.12)$ & $(6.75)$ & $(7.45)$ & $(8.26)$ & $(8.86)$ & $(9.44)$ & $(10.45)$ & $(10.92)$ & (11.19) & (11.60) & (11.90) & $(12.25)$ & (11.38) & $(10.22)$ & $(8.75)$ & $(6.82)$ & $(9.68)$ \\
\hline \multirow[t]{2}{*}{ BDN-2010-1 } & 5.67 & 8.00 & 12.67 & 17.00 & 18.67 & 20.67 & 22.67 & 26.67 & 29.67 & 32.00 & 36.00 & 45.00 & 39.00 & 20.67 & 14.00 & 8.00 & 22.27 \\
\hline & $(2.48)$ & $(2.92)$ & $(3.63)$ & $(4.18)$ & $(4.38)$ & $(4.60)$ & $(4.81)$ & $(5.21)$ & $(5.49)$ & $(5.70)$ & $(6.04)$ & $(6.75)$ & $(6.28)$ & $(4.60)$ & $(3.81)$ & $(2.92)$ & $(4.77)$ \\
\hline \multirow[t]{2}{*}{ BDN-2013-41 } & 14.67 & 21.00 & 30.33 & 37.00 & 41.67 & 54.67 & 70.67 & 78.67 & 76.67 & 89.00 & 99.67 & 111.67 & 101.00 & 38.67 & 25.67 & 19.67 & 56.92 \\
\hline & $(3.89)$ & $(4.64)$ & $(5.55)$ & $(6.12)$ & $(6.49)$ & $(7.43)$ & $(8.44)$ & $(8.90)$ & $(8.78)$ & $(9.46)$ & $(10.01)$ & (10.59) & (10.07) & $(6.26)$ & $(5.12)$ & $(4.49)$ & $(7.58)$ \\
\hline \multirow[t]{2}{*}{ BDN-2014-1 } & 15.00 & 31.67 & 36.67 & 43.00 & 57.00 & 64.00 & 81.67 & 93.00 & 112.67 & 119.67 & 125.67 & 149.67 & 147.00 & 134.67 & 96.67 & 64.67 & 85.79 \\
\hline & $(3.94)$ & $(5.67)$ & $(6.10)$ & $(6.60)$ & $(7.58)$ & $(8.03)$ & $(9.06)$ & $(9.67)$ & $(10.64)$ & (10.96) & $(11.23)$ & (12.25) & (12.14) & (11.63) & $(9.86)$ & $(8.07)$ & $(9.29)$ \\
\hline \multirow[t]{2}{*}{ BDN-2014-3 } & 27.67 & 30.00 & 37.00 & 42.00 & 44.00 & 49.67 & 57.67 & 64.00 & 75.67 & 81.67 & 85.67 & 97.67 & 68.00 & 54.00 & 38.67 & 32.00 & 55.33 \\
\hline & $(5.31)$ & $(5.52)$ & $(6.12)$ & $(6.52)$ & $(6.67)$ & (7.08) & (7.63) & $(8.03)$ & $(8.73)$ & $(9.06)$ & $(9.28)$ & $(9.91)$ & $(8.28)$ & $(7.38)$ & $(6.26)$ & $(5.70)$ & (7.47) \\
\hline \multirow[t]{2}{*}{ BSMR-736 } & 31.00 & 43.67 & 48.00 & 57.67 & 68.00 & 74.00 & 79.67 & 83.67 & 90.67 & 94.00 & 100.00 & 109.67 & 100.67 & 87.33 & 67.67 & 43.00 & 73.67 \\
\hline & $(5.61)$ & $(6.65)$ & (6.96) & (7.63) & $(8.28)$ & $(8.63)$ & $(8.95)$ & $(9.17)$ & $(9.55)$ & $(9.72)$ & $(10.02)$ & (10.50) & (10.06) & (9.37) & $(8.26)$ & $(6.60)$ & $(8.61)$ \\
\hline \multirow[t]{2}{*}{ BSMR-846 } & 63.67 & 74.00 & 87.00 & 99.67 & 112.67 & 122.67 & 119.67 & 127.67 & 136.00 & 139.00 & 150.00 & 164.00 & 126.00 & 110.00 & 88.67 & 72.67 & 112.08 \\
\hline & $(8.01)$ & $(8.63)$ & $(9.35)$ & (10.01) & (10.64) & (11.10) & (10.96) & (11.32) & (11.68) & (11.81) & (12.27) & (12.83) & $(11.25)$ & $(10.51)$ & $(9.44)$ & $(8.55)$ & $(10.61)$ \\
\hline \multirow[t]{2}{*}{ KALITUR } & 14.00 & 21.00 & 25.33 & 33.00 & 40.67 & 45.67 & 57.67 & 62.67 & 66.00 & 71.00 & 84.00 & 95.00 & 82.00 & 55.33 & 44.00 & 35.00 & 52.02 \\
\hline & $(3.81)$ & $(4.64)$ & $(5.08)$ & (5.79) & $(6.42)$ & (6.79) & (7.63) & (7.95) & $(8.15)$ & $(8.46)$ & (9.19) & $(9.77)$ & $(9.08)$ & $(7.47)$ & $(6.67)$ & $(5.96)$ & $(7.25)$ \\
\hline \multirow[t]{2}{*}{ KHADKI } & 46.00 & 42.00 & 62.00 & 77.67 & 85.00 & 92.67 & 107.00 & 114.00 & 124.00 & 127.50 & 142.00 & 166.67 & 122.00 & 109.33 & 88.00 & 58.00 & 97.74 \\
\hline & $(6.82)$ & $(6.52)$ & $(7.91)$ & $(8.84)$ & $(9.25)$ & $(9.65)$ & (10.37) & (10.70) & (11.16) & (11.31) & (11.94) & (12.93) & (11.07) & (10.48) & $(9.41)$ & $(7.65)$ & $(9.91)$ \\
\hline \multirow[t]{2}{*}{ GULYAL } & 14.67 & 26.67 & 37.00 & 44.67 & 55.00 & 61.67 & 67.00 & 75.67 & 88.00 & 96.00 & 109.00 & 116.67 & 102.67 & 91.00 & 79.00 & 45.67 & 69.40 \\
\hline & $(3.89)$ & $(5.21)$ & $(6.12)$ & $(6.72)$ & $(7.45)$ & (7.88) & $(8.22)$ & $(8.73)$ & $(9.41)$ & $(9.82)$ & (10.46) & (10.82) & (10.16) & $(9.57)$ & $(8.92)$ & $(6.79)$ & $(8.36)$ \\
\hline \multirow[t]{2}{*}{ BRG-1 } & 99.67 & 162.00 & 178.33 & 200.67 & 187.67 & 211.00 & 214.00 & 223.00 & 237.00 & 251.67 & 255.00 & 261.00 & 224.67 & 214.00 & 200.00 & 186.00 & 206.60 \\
\hline & (10.01) & $(12.75)$ & (13.37) & (14.18) & $(13.72)$ & (14.54) & $(14.65)$ & (14.95) & $(15.41)$ & (15.88) & (15.98) & (16.17) & (15.01) & $(14.65)$ & (14.16) & $(13.66)$ & $(14.39)$ \\
\hline \multirow[t]{2}{*}{ BRG-2 } & 214.00 & 243.33 & 274.50 & 281.00 & 299.50 & 284.67 & 280.50 & 288.00 & 282.67 & 290.50 & 305.00 & 306.67 & 280.67 & 286.67 & 267.50 & 257.00 & 277.64 \\
\hline & (14.65) & $(15.62)$ & (16.58) & (16.78) & $(17.32)$ & (16.89) & (16.76) & (16.99) & (16.83) & (17.06) & (17.48) & (17.53) & (16.77) & $(16.95)$ & (16.37) & $(16.05)$ & $(16.68)$ \\
\hline
\end{tabular}




\begin{tabular}{|c|c|c|c|c|c|c|c|c|c|c|c|c|c|c|c|c|c|}
\hline \multirow[t]{2}{*}{ LRG-41 } & 36.67 & 52.67 & 62.00 & 75.00 & 87.67 & 91.67 & 107.00 & 126.67 & 130.67 & 136.00 & 138.67 & 148.67 & 132.00 & 116.67 & 96.67 & 57.00 & 99.73 \\
\hline & (6.10) & $(7.29)$ & $(7.91)$ & (8.69) & (9.39) & $(9.60)$ & (10.37) & (11.28) & (11.45) & (11.68) & (11.80) & (12.21) & (11.51) & (10.82) & $(9.86)$ & (7.58) & (10.01) \\
\hline \multirow[t]{2}{*}{ ICP-7035 } & 112.67 & 134.00 & 153.00 & 178.00 & 195.67 & 183.00 & 198.00 & 216.67 & 223.67 & 232.67 & 238.67 & 248.00 & 221.67 & 180.00 & 156.00 & 120.67 & 187.02 \\
\hline & (10.64) & (11.60) & (12.39) & (13.36) & (14.01) & (13.55) & (14.09) & (14.74) & (14.97) & (15.27) & (15.47) & (15.76) & (14.91) & (13.44) & (12.51) & (11.01) & (13.69) \\
\hline \multirow[t]{2}{*}{ ICP-10531 } & 17.67 & 23.67 & 30.00 & 34.67 & 40.00 & 38.67 & 39.00 & 41.67 & 45.00 & 50.67 & 51.67 & 56.50 & 36.00 & 27.67 & 21.67 & 18.00 & 35.78 \\
\hline & $(4.26)$ & $(4.92)$ & $(5.52)$ & (5.93) & (6.36) & $(6.26)$ & (6.28) & $(6.49)$ & $(6.75)$ & (7.15) & $(7.22)$ & (7.55) & (6.04) & (5.31) & $(4.71)$ & $(4.30)$ & (6.02) \\
\hline \multirow[t]{2}{*}{ ICPL-322 } & 43.67 & 52.00 & 61.00 & 66.00 & 69.67 & 74.00 & 77.00 & 81.00 & 88.67 & 94.00 & 96.00 & 100.67 & 85.00 & 73.67 & 63.00 & 50.67 & 73.50 \\
\hline & $(6.65)$ & $(7.25)$ & $(7.84)$ & $(8.15)$ & (8.38) & (8.63) & $(8.80)$ & $(9.03)$ & $(9.44)$ & $(9.72)$ & $(9.82)$ & (10.06) & $(9.25)$ & $(8.61)$ & $(7.97)$ & (7.15) & $(8.60)$ \\
\hline \multirow[t]{2}{*}{ BSR-1 } & 12.00 & 15.67 & 18.00 & 25.00 & 27.00 & 30.00 & 31.67 & 35.67 & 39.67 & 40.67 & 43.00 & 45.00 & 32.00 & 26.00 & 20.00 & 14.00 & 28.46 \\
\hline & $(3.54)$ & $(4.02)$ & $(4.30)$ & $(5.05)$ & $(5.24)$ & $(5.52)$ & $(5.67)$ & $(6.01)$ & $(6.34)$ & $(6.42)$ & $(6.60)$ & $(6.75)$ & $(5.70)$ & $(5.15)$ & $(4.53)$ & (3.81) & $(5.38)$ \\
\hline \multirow[t]{2}{*}{ V-127 } & 3.00 & 3.67 & 4.33 & 6.00 & 6.67 & 8.67 & 13.00 & 14.00 & 13.67 & 16.67 & 18.67 & 22.67 & 16.00 & 13.00 & 3.67 & 2.00 & 10.35 \\
\hline & (1.87) & $(2.04)$ & $(2.20)$ & $(2.55)$ & (2.68) & $(3.03)$ & (3.67) & $(3.81)$ & $(3.76)$ & $(4.14)$ & $(4.38)$ & $(4.81)$ & $(4.06)$ & $(3.67)$ & $(2.04)$ & (1.58) & $(3.29)$ \\
\hline \multirow{2}{*}{$\begin{array}{l}\text { Cajanus } \\
\text { cajanifolius }\end{array}$} & 0.00 & 0.00 & 0.00 & 0.00 & 0.00 & 0.00 & 0.00 & 0.00 & 0.00 & 5.67 & 8.67 & 20.67 & 7.00 & 10.00 & 1.67 & 0.00 & 3.98 \\
\hline & $(0.71)$ & $(0.71)$ & $(0.71)$ & $(0.71)$ & $(0.71)$ & $(0.71)$ & $(0.71)$ & $(0.71)$ & $(0.71)$ & $(2.48)$ & (3.03) & $(4.60)$ & (4.18) & $(3.24)$ & $(1.47)$ & $(0.71)$ & $(2.12)$ \\
\hline \multirow{2}{*}{$\begin{array}{l}\text { Cajanus } \\
\text { scarabaeoides }\end{array}$} & 0.00 & 0.00 & 0.00 & 0.00 & 0.00 & 0.00 & 0.00 & 0.00 & 0.00 & 0.00 & 0.00 & 0.00 & 0.00 & 0.00 & 0.00 & 0.00 & 0.00 \\
\hline & $(0.71)$ & $(0.71)$ & $(0.71)$ & $(0.71)$ & $(0.71)$ & $(0.71)$ & $(0.71)$ & $(0.71)$ & $(0.71)$ & $(0.71)$ & $(0.71)$ & $(0.71)$ & $(0.71)$ & $(0.71)$ & $(0.71)$ & $(0.71)$ & $(0.71)$ \\
\hline Average & 39.87 & 51.50 & 60.61 & 69.28 & 75.73 & 79.80 & 86.63 & 93.57 & 99.25 & 105.12 & 111.42 & 120.78 & 103.12 & 87.63 & 72.43 & 56.50 & 82.08 \\
\hline $\mathrm{SE}(\mathrm{m}) \pm$ & 0.24 & 0.19 & 0.19 & 0.21 & 0.25 & 0.16 & 0.14 & 0.15 & 0.20 & 0.18 & 0.16 & 0.15 & 0.17 & 0.17 & 0.22 & 0.26 & 0.13 \\
\hline CD at $5 \%$ & 0.69 & 0.55 & 0.53 & 0.61 & 0.71 & 0.46 & 0.41 & 0.43 & 0.57 & 0.52 & 0.45 & 0.44 & 0.48 & 0.50 & 0.62 & 0.74 & 0.36 \\
\hline CV \% & 7.72 & 5.35 & 4.76 & 5.06 & 5.59 & 3.50 & 2.95 & 2.98 & 3.79 & 3.35 & 2.78 & 2.62 & 3.10 & 3.54 & 5.00 & 6.90 & 2.68 \\
\hline
\end{tabular}

Figures of population in parenthesis are $\sqrt{x}+0.5$ transformed values.

Table.2 M. obtusa (Malloch) pupal population in different pigeonpea genotypes

\begin{tabular}{|c|c|c|c|c|c|c|c|c|c|c|c|c|c|c|c|c|c|}
\hline \multirow[t]{2}{*}{ GENOTYPE } & \multicolumn{17}{|c|}{ Pupal Population per 100 pods per SMW } \\
\hline & 44 & 45 & 46 & 47 & 48 & 49 & 50 & 51 & 52 & 01 & 02 & 03 & 04 & 05 & 06 & 07 & Mean \\
\hline BDN-2 & $\begin{array}{c}7.67 \\
(2.86)\end{array}$ & $\begin{array}{l}12.67 \\
(3.63)\end{array}$ & $\begin{array}{l}16.67 \\
(4.14)\end{array}$ & $\begin{array}{l}20.67 \\
(4.60)\end{array}$ & $\begin{array}{l}22.67 \\
(4.81)\end{array}$ & $\begin{array}{l}28.67 \\
(5.40)\end{array}$ & $\begin{array}{l}34.00 \\
(5.87)\end{array}$ & $\begin{array}{l}42.00 \\
(6.52)\end{array}$ & $\begin{array}{l}46.00 \\
(6.82)\end{array}$ & $\begin{array}{l}50.00 \\
(7.11)\end{array}$ & $\begin{array}{l}60.00 \\
(7.78)\end{array}$ & $\begin{array}{l}63.67 \\
(8.01)\end{array}$ & $\begin{array}{l}55.67 \\
(7.49)\end{array}$ & $\begin{array}{l}35.67 \\
(6.01)\end{array}$ & $\begin{array}{l}22.67 \\
(4.81)\end{array}$ & $\begin{array}{l}12.67 \\
(3.63)\end{array}$ & $\begin{array}{l}33.21 \\
(5.81)\end{array}$ \\
\hline BDN-2010-1 & $\begin{array}{c}3.00 \\
(1.87)\end{array}$ & $\begin{array}{c}5.67 \\
(2.48)\end{array}$ & $\begin{array}{c}5.00 \\
(2.35)\end{array}$ & $\begin{array}{c}4.00 \\
(2.12)\end{array}$ & $\begin{array}{c}7.00 \\
(2.74)\end{array}$ & $\begin{array}{c}7.00 \\
(2.74)\end{array}$ & $\begin{array}{c}8.00 \\
(2.92)\end{array}$ & $\begin{array}{c}6.00 \\
(2.55)\end{array}$ & $\begin{array}{c}8.00 \\
(2.92)\end{array}$ & $\begin{array}{l}11.00 \\
(3.39)\end{array}$ & $\begin{array}{l}16.00 \\
(4.06)\end{array}$ & $\begin{array}{l}24.67 \\
(5.02)\end{array}$ & $\begin{array}{c}10.00 \\
(3.24)\end{array}$ & $\begin{array}{l}10.00 \\
(3.24)\end{array}$ & $\begin{array}{c}10.67 \\
(3.34)\end{array}$ & $\begin{array}{c}8.00 \\
(2.92)\end{array}$ & $\begin{array}{c}9.00 \\
(3.08)\end{array}$ \\
\hline
\end{tabular}




\begin{tabular}{|c|c|c|c|c|c|c|c|c|c|c|c|c|c|c|c|c|c|}
\hline \multirow[t]{2}{*}{ BDN-2013-41 } & 5.00 & 7.67 & 11.00 & 13.00 & 14.67 & 19.67 & 26.00 & 28.00 & 27.67 & 32.00 & 36.67 & 42.67 & 36.00 & 14.67 & 10.00 & 7.00 & 20.73 \\
\hline & $(2.35)$ & $(2.86)$ & $(3.39)$ & (3.67) & $(3.89)$ & $(4.49)$ & $(5.15)$ & (5.34) & $(5.31)$ & $(5.70)$ & (6.10) & $(6.57)$ & $(6.04)$ & $(3.89)$ & (3.24) & (2.74) & $(4.61)$ \\
\hline \multirow[t]{2}{*}{ BDN-2014-1 } & 6.67 & 13.67 & 15.33 & 19.67 & 24.67 & 28.67 & 34.00 & 42.00 & 50.67 & 54.00 & 56.67 & 57.00 & 61.67 & 49.00 & 44.00 & 29.00 & 36.67 \\
\hline & (2.68) & $(3.76)$ & $(3.98)$ & $(4.49)$ & $(5.02)$ & $(5.40)$ & $(5.87)$ & $(6.52)$ & (7.15) & (7.38) & $(7.56)$ & (7.58) & (7.88) & (7.04) & $(6.67)$ & $(5.43)$ & $(6.10)$ \\
\hline \multirow[t]{2}{*}{ BDN-2014-3 } & 11.67 & 16.00 & 18.00 & 19.67 & 22.00 & 27.67 & 31.50 & 34.67 & 40.00 & 44.67 & 48.67 & 58.00 & 45.00 & 34.00 & 27.67 & 22.67 & 31.36 \\
\hline & $(3.49)$ & $(4.06)$ & $(4.30)$ & $(4.49)$ & $(4.74)$ & $(5.31)$ & $(5.66)$ & $(5.93)$ & (6.36) & $(6.72)$ & $(7.01)$ & $(7.65)$ & $(6.75)$ & $(5.87)$ & $(5.31)$ & $(4.81)$ & $(5.64)$ \\
\hline \multirow[t]{2}{*}{ BSMR-736 } & 10.67 & 15.67 & 17.67 & 21.00 & 23.00 & 26.00 & 28.00 & 29.00 & 32.67 & 35.67 & 39.67 & 49.00 & 39.00 & 32.00 & 24.00 & 16.67 & 27.48 \\
\hline & (3.34) & $(4.02)$ & $(4.26)$ & $(4.64)$ & $(4.85)$ & $(5.15)$ & $(5.34)$ & $(5.43)$ & (5.76) & $(6.01)$ & $(6.34)$ & (7.04) & $(6.28)$ & $(5.70)$ & $(4.95)$ & (4.14) & $(5.29)$ \\
\hline \multirow[t]{2}{*}{ BSMR-846 } & 13.67 & 19.00 & 22.67 & 26.67 & 29.67 & 32.00 & 45.50 & 49.00 & 51.67 & 53.67 & 57.67 & 68.67 & 60.67 & 53.00 & 42.67 & 35.67 & 41.36 \\
\hline & (3.76) & $(4.42)$ & $(4.81)$ & $(5.21)$ & $(5.49)$ & $(5.70)$ & $(6.78)$ & (7.04) & $(7.22)$ & $(7.36)$ & (7.63) & $(8.32)$ & $(7.82)$ & (7.31) & $(6.57)$ & $(6.01)$ & $(6.47)$ \\
\hline \multirow[t]{2}{*}{ KALITUR } & 7.00 & 12.00 & 15.00 & 16.00 & 19.67 & 21.67 & 25.50 & 28.67 & 30.00 & 33.67 & 39.00 & 42.67 & 38.67 & 32.67 & 26.00 & 23.67 & 25.74 \\
\hline & (2.74) & $(3.54)$ & (3.94) & (4.06) & $(4.49)$ & $(4.71)$ & $(5.10)$ & $(5.40)$ & $(5.52)$ & $(5.85)$ & (6.28) & $(6.57)$ & $(6.26)$ & $(5.76)$ & $(5.15)$ & (4.92) & $(5.12)$ \\
\hline \multirow[t]{2}{*}{ KHADKI } & 6.00 & 12.00 & 16.67 & 21.67 & 25.00 & 30.67 & 35.00 & 47.67 & 51.00 & 54.67 & 60.00 & 69.67 & 59.67 & 54.67 & 49.00 & 38.00 & 39.46 \\
\hline & $(2.55)$ & $(3.54)$ & $(4.14)$ & $(4.71)$ & $(5.05)$ & $(5.58)$ & $(5.96)$ & $(6.94)$ & (7.18) & $(7.43)$ & $(7.78)$ & $(8.38)$ & $(7.76)$ & $(7.43)$ & $(7.04)$ & $(6.20)$ & $(6.32)$ \\
\hline \multirow[t]{2}{*}{ GULYAL } & 3.67 & 6.67 & 9.00 & 11.67 & 14.00 & 21.67 & 23.50 & 26.67 & 30.67 & 34.00 & 56.67 & 60.67 & 52.00 & 47.00 & 40.67 & 23.67 & 28.89 \\
\hline & (2.04) & $(2.68)$ & (3.08) & (3.49) & $(3.81)$ & $(4.71)$ & $(4.90)$ & (5.21) & (5.58) & (5.87) & $(7.56)$ & (7.82) & $(7.25)$ & (6.89) & $(6.42)$ & (4.92) & (5.42) \\
\hline \multirow[t]{2}{*}{ BRG-1 } & 26.67 & 31.67 & 47.67 & 53.00 & 50.67 & 83.00 & 84.50 & 88.00 & 93.67 & 99.00 & 116.00 & 123.00 & 111.67 & 106.67 & 101.67 & 94.00 & 81.93 \\
\hline & $(5.21)$ & $(5.67)$ & $(6.94)$ & $(7.31)$ & (7.15) & $(9.14)$ & $(9.22)$ & $(9.41)$ & (9.70) & $(9.97)$ & (10.79) & (11.11) & (10.59) & (10.35) & (10.11) & $(9.72)$ & (9.08) \\
\hline \multirow[t]{2}{*}{ BRG-2 } & 45.67 & 52.00 & 63.67 & 62.00 & 64.67 & 98.33 & 98.50 & 102.00 & 100.00 & 108.67 & 143.67 & 138.67 & 138.67 & 136.00 & 135.67 & 132.00 & 101.26 \\
\hline & (6.79) & $(7.25)$ & $(8.01)$ & (7.91) & $(8.07)$ & $(9.94)$ & $(9.95)$ & $(10.12)$ & (10.02) & (10.45) & (12.01) & (11.80) & (11.80) & (11.68) & (11.67) & (11.51) & (10.09) \\
\hline \multirow[t]{2}{*}{ LRG-41 } & 9.67 & 12.67 & 14.67 & 18.00 & 20.67 & 30.00 & 37.50 & 44.67 & 48.67 & 52.00 & 71.67 & 76.00 & 69.00 & 65.00 & 50.00 & 28.67 & 40.55 \\
\hline & (3.19) & (3.63) & (3.89) & $(4.30)$ & $(4.60)$ & $(5.52)$ & $(6.16)$ & (6.72) & (7.01) & $(7.25)$ & $(8.50)$ & $(8.75)$ & (8.34) & (8.09) & (7.11) & (5.40) & (6.41) \\
\hline ICP-7035 & 32.00 & 38.00 & 44.00 & 50.67 & 55.67 & 64.00 & 84.00 & 89.67 & 92.67 & 97.67 & 99.67 & 123.67 & 114.67 & 94.67 & 81.67 & 61.67 & 76.52 \\
\hline
\end{tabular}




\begin{tabular}{|c|c|c|c|c|c|c|c|c|c|c|c|c|c|c|c|c|c|}
\hline & $(5.70)$ & $(6.20)$ & (6.67) & $(7.15)$ & $(7.49)$ & $(8.03)$ & (9.19) & $(9.50)$ & $(9.65)$ & $(9.91)$ & (10.01) & (11.14) & (10.73) & (9.76) & $(9.06)$ & (7.88) & $(8.78)$ \\
\hline \multirow[t]{2}{*}{ ICP-10531 } & 5.00 & 7.67 & 10.00 & 12.00 & 13.67 & 14.67 & 17.00 & 16.67 & 18.67 & 19.67 & 23.67 & 25.67 & 16.67 & 12.67 & 10.00 & 8.67 & 14.52 \\
\hline & $(2.35)$ & $(2.86)$ & (3.24) & (3.54) & (3.76) & (3.89) & (4.18) & $(4.14)$ & (4.38) & $(4.49)$ & $(4.92)$ & (5.12) & (4.14) & (3.63) & (3.24) & (3.03) & (3.88) \\
\hline \multirow[t]{2}{*}{ ICPL-322 } & 12.67 & 15.00 & 18.00 & 19.67 & 20.67 & 27.67 & 30.00 & 31.67 & 35.67 & 40.67 & 51.00 & 54.67 & 44.67 & 37.67 & 34.00 & 27.00 & 31.29 \\
\hline & (3.63) & (3.94) & $(4.30)$ & (4.49) & $(4.60)$ & $(5.31)$ & $(5.52)$ & (5.67) & (6.01) & $(6.42)$ & (7.18) & (7.43) & $(6.72)$ & (6.18) & $(5.87)$ & (5.24) & $(5.64)$ \\
\hline \multirow[t]{2}{*}{ BSR-1 } & 0.00 & 0.00 & 0.67 & 5.67 & 8.00 & 8.00 & 10.00 & 11.00 & 9.67 & 11.00 & 10.67 & 12.67 & 16.00 & 14.00 & 7.67 & 5.00 & 8.13 \\
\hline & $(0.71)$ & $(0.71)$ & (1.08) & $(2.48)$ & (2.92) & $(2.92)$ & (3.24) & (3.39) & (3.19) & (3.39) & (3.34) & (3.63) & (4.06) & (3.81) & $(2.86)$ & (2.35) & (2.94) \\
\hline \multirow[t]{2}{*}{ V-127 } & 0.00 & 0.00 & 0.00 & 1.00 & 1.00 & 1.67 & 4.00 & 5.67 & 5.00 & 6.67 & 6.67 & 6.00 & 3.67 & 2.00 & 6.00 & 4.67 & 3.38 \\
\hline & $(0.71)$ & $(0.71)$ & $(0.71)$ & $(1.22)$ & $(1.22)$ & (1.47) & (2.12) & $(2.48)$ & $(2.35)$ & $(2.68)$ & (2.68) & (2.55) & (2.04) & (1.58) & (2.55) & (2.27) & (1.97) \\
\hline \multirow{2}{*}{$\begin{array}{l}\text { Cajanus } \\
\text { cajanifolius }\end{array}$} & 0.00 & 0.00 & 0.00 & 0.00 & 0.00 & 0.00 & 0.00 & 0.00 & 0.00 & 0.00 & 1.67 & 4.00 & 4.00 & 1.67 & 0.67 & 0.00 & 0.75 \\
\hline & $(0.71)$ & $(0.71)$ & $(0.71)$ & $(0.71)$ & $(0.71)$ & $(0.71)$ & $(0.71)$ & $(0.71)$ & $(0.71)$ & $(0.71)$ & (1.47) & $(2.12)$ & $(2.12)$ & (1.47) & (1.08) & $(0.71)$ & (1.12) \\
\hline \multirow{2}{*}{$\begin{array}{l}\text { Cajanus } \\
\text { scarabaeoides }\end{array}$} & 0.00 & 0.00 & 0.00 & 0.00 & 0.00 & 0.00 & 0.00 & 0.00 & 0.00 & 0.00 & 0.00 & 0.00 & 0.00 & 0.00 & 0.00 & 0.00 & 0.00 \\
\hline & $(0.71)$ & $(0.71)$ & $(0.71)$ & $(0.71)$ & $(0.71)$ & $(0.71)$ & $(0.71)$ & $(0.71)$ & $(0.71)$ & $(0.71)$ & $(0.71)$ & $(0.71)$ & $(0.71)$ & $(0.71)$ & $(0.71)$ & $(0.71)$ & $(0.71)$ \\
\hline \multirow{4}{*}{$\begin{array}{l}\text { Average } \\
\text { SE (m) } \pm \\
\text { CD at } 5 \% \\
\text { CV \% }\end{array}$} & 10.33 & 13.90 & 17.28 & 19.80 & 21.87 & 28.55 & 32.83 & 36.15 & 38.62 & 41.93 & 49.78 & 55.05 & 48.87 & 41.65 & 36.23 & 28.93 & 32.61 \\
\hline & 0.19 & 0.16 & 0.11 & 0.13 & 0.16 & 0.18 & 0.18 & 0.15 & 0.18 & 0.14 & 0.16 & 0.17 & 0.14 & 0.17 & 0.25 & 0.26 & 0.11 \\
\hline & 0.54 & 0.45 & 0.31 & 0.39 & 0.47 & 0.51 & 0.51 & 0.44 & 0.52 & 0.41 & 0.45 & 0.48 & 0.40 & 0.48 & 0.72 & 0.73 & 0.31 \\
\hline & 11.55 & 8.18 & 5.08 & 5.75 & 6.58 & 6.45 & 5.87 & 4.84 & 5.51 & 4.19 & 4.19 & 4.24 & 3.75 & 4.96 & 8.12 & 9.43 & 3.64 \\
\hline
\end{tabular}

Figures of population in parenthesis are $\sqrt{x}+0.5$ transformed values. 
The population in various standard weeks was found in order $9^{\text {th }}>8^{\text {th }}>10^{\text {th }}>7^{\text {th }}>11^{\text {th }}>6^{\text {th }}>$ $12^{\text {th }}>5^{\text {th }}>4^{\text {th }}$. The highest mean population of pod fly was recorded in NDA-5-25 (0.57 maggots per 10 pods), followed by MAL-20 (0.46 maggots per 10 pods), PDA $85-5 \mathrm{E}$ (0.33 maggots per 10 pods), MAL-13 (0.31 maggots per 10 plots), MAL- $27 \quad(0.28$ maggots per 10 pods)and the lowest in KAWR 92-2 (0.21 maggots per 10 pods). Similarly, Revathi et al., (2015) found a variation among different pigeonpea genotypes with respect to number of maggots ranging from zero to four per pod, whereas the genotype, 2011-5 recorded highest number of maggots per pod i.e., 1.5 and the genotype ENT-11 recorded least number of maggots (0.5), respectively. Patange et al., (2016) also reported that the pod fly population ranged from 0.81 per plant in BSMR-736 to 1.02 per plant in BDN-708.

The data on pupal population of M. obtusa on 20 pigeonpea genotypes under study during $44^{\text {th }}$ SMW to $07^{\text {th }}$ SMW is presented in Table 2. It is clear from the data that all genotypes indicated significant differences regarding pupal population of pigeonpea pod fly, $M$. obtusa. The mean pupal population of $M$. obtusa varied from 0.67 to 143.67 pupae per 100 pods during crop season (i.e. $44^{\text {th }}$ to $07^{\text {th }}$ SMW). The pupae of $M$. obtusa were observed first in $44^{\text {th }}$ SMW and were recorded till $07^{\text {th }}$ SMW in maximum pigeonpea genotypes. The pupal population of M. obtusa was ranging from 7.67 to 63.67 pupae per 100 pods in BDN-2, 3.00 to 24.67 pupae per 100 pods in BDN-2010-1, 5.00 to 42.67 pupae per 100 pods in BDN-2013-41, 6.67 to 61.67 pupae per 100 pods in BDN-2014-1, 11.67 to 58.00 pupae per 100 pods in BDN-2014-3, 10.67 to 49.00 pupae per 100 pods in BSMR$736,13.67$ to 68.67 pupae per 100 pods in BSMR-846, 7.00 to 42.67 pupae per 100 pods in KALITUR, 6.00 to 69.67 pupae per 100 pods in KHADKI, 3.67 to 60.67 pupae per
100 pods in GULYAL, 26.67 to 123.00 pupae per 100 pods in BRG-1, 45.67 to 138.67 pupae per 100 pods in BRG-2, 9.67 to 76.00 pupae per 100 pods in LRG-41, 32.00 to 123.67 pupae per 100 pods in ICP-7035, 5.00 to 25.67 pupae per 100 pods in ICP-10531, 12.67 to 54.67 pupae per 100 pods in ICP$322,0.00$ to 16.00 pupae per 100 pods in BSR-1, 0.00 to 6.67 pupae per 100 pods in V127 and 0.00 to 4.00 pupae per 100 pods in $C$. cajanifolius, respectively, while, no pupa of the $M$. obtusa was observed in $C$. scarabaeoides, indicating high resistance against $M$. obtusa. The peak pupal population of pod fly irrespective of genotype was recorded in $03^{\text {rd }}$ SMW and thereafter, it declined due to maturity of grains and reached to minimum on $07^{\text {th }}$ SMW in all the genotypes. The pupal population of $M$. obtusa on different pigeonpea genotypes in descending order was $03^{\text {rd }}>02^{\text {nd }}>04^{\text {th }}>01^{\text {st }}>$ $05^{\text {th }}>52^{\text {nd }}>51^{\text {st }}>06^{\text {th }}>50^{\text {th }}>49^{\text {th }}>07^{\text {th }}>48^{\text {th }}>$ $47^{\text {th }}>46^{\text {th }}>45^{\text {th }}>44^{\text {th }}$ SMW during the crop period.

The highest pupal population of $M$. obtusa (Malloch) was recorded in BRG-2 (101.26 pupae per 100 pods), followed by BRG-1 (81.93 pupae per 100 pods), ICP-7035 (76.52 pupae per 100 pods), BSMR-846 (41.36 pupae per 100 pods), LRG-41 (40.55 pupae per 100 pods), KHADKI (39.46 pupae per 100 pods) and BDN-2014-1 (36.67 pupae per 100 pods), respectively. The lowest pupal population of $M$. obtusa (Malloch) was recorded in the genotype, $C$. scarabaeoides (no pupa), C. cajanifolius ( 0.75 pupae per 100 pods), followed by V-127 (3.38 pupae per 100 pods), BSR-1 (8.13 pupae per 100 pods), BDN-2010-1 (9.00 pupae per 100 pods) and ICP-10531 (14.52 pupae per 100 pods), respectively, while, the genotypes, $\mathrm{BDN}$ 2013-41 (20.73 pupae per 100 pods), KALITUR (25.74 pupae per 100 pods), BSMR-736 (27.48 pupae per 100 pods), GULYAL (28.89 pupae per 100 pods), BDN- 
2 (33.21 pupae per 100 pods), BDN-2014-3 (31.36 pupae per 100 pods) and ICPL-322 (31.29 pupae per 100 pods), respectively shown intermittent pupal population of $M$. obtusa.

The present findings are in accordance with the reports of Sharma et al., (2003), who reported that accessions belonging to Cajanus scarabaeoides (L.) Thouars showed resistance against pod fly. Revathi et al.,(2015)found the variation among different pigeonpea genotypes with respect to number of pupae ranging from $0-6$ per pod, where, the genotype, 2011-5 recorded highest number of pupae per pod i.e., 1.7 while, the genotype WRG-51 recorded least number of pupae (0.5) per pod. Similarly, Patange et al., (2016) reported that pod fly population ranged from 0.81 per plant in BSMR-736 to 1.02 per plant in BDN-708.

\section{References}

Chiranjeevi, B. and Patange, N.R. 2017. New host record of three hymenopteran parasitoids associated with Melanagromyza obtusa (Malloch) and their level of natural parasitism. Journal of Entomology and Zoology Studies. 5(4): 277-283.

Ipe. M. Ipe. 1974. Morphological behavioural and biological studies of Melanagromyza obtusa (Malloch) (Diptera: Agromyzidae) on Cajanus indicus Spreng. Zeitschrift fuer Angewandte Entomologie. 75: 89-98.

Keval, R., Kerketta, D., Nath, P. and Singh, P.S. 2010. Population fluctuations of pod fly on some varieties of pigeonpea. Journal of Food Legumes. 23(2): 164165.

Kumar, A.L. and Nath, P. 2003. Pest complex and their population dynamics on medium-late variety of pigeonpea
Bahar. Indian Journal of Pulses Research.16: 150-154.

Kumar, S., Singh, B. and Kumar, N. 2003. Assessment of pod damage caused by pod borere complex in pre-rabi pigeonpea. Indian Journal of Pulses Research.16: 169-170.

Lateef, S.S. and Reed, W. 1990. Insect pests on pigeonpea. In: S.R. Singh (Ed.), Insect Pests of Tropical Food Legumes, John Wiley and Sons, New York. pp. 193-242.

Nath, P., Singh, R.S., Singh, P.S. and Keval, R. 2008. Study of the succession of insect pest associated with pods of pigeonpea under sole and intercropping system. Indian Journal of Environment and Ecoplan.15: 455-461.

Patange, N.R., Chiranjeevi, B., Patil, D.K. and Gite, V.K. 2016. Evaluation of pigeonpea genotypes under severe biotic stress of pod borer complex. In: Proceedings of National Seminar on "Breeding of Field Crops for Biotic and Abiotic Stress in Relation to Climate Change", 28-29 March 2016, held at College of Agriculture, VNMKV, Parbhani (MS), India. pp. 221.

Revathi, K., Sreekanth, M., Krishnayya, P.V. and Rao, V.S. 2015. Incidence of pod fly, Melanagromyza obtusa (Malloch) and its influence on weight loss in different pigeonpea genotypes. International Journal of Innovative Science, Engineering and Technology. 2(5): 460-464.

Shanower, T.G., Romeis, J. and Minja, E.M. 1999. Insect pests of pigeonpea and their management. Annual Review of Entomology. 44: 77-96.

Shanower, T.G., Lal, S.S. and Bhagwat, V.R. 1998. Biology and management of Melanagromyza obtusa (Malloch) (Diptera: Agromyzidae). Crop Protection. 17(3): 249-263. 
Sharma, H.C., Pampapathy, G. and Reddy, L.J. 2003. Wild relatives of pigeonpea as a source of resistance to the pod fly, Melanagromyza obtusa (Malloch) and pod wasp (Tanaostigmodes cajaninae La Salle). Genetic Resources and Crop Evolution. 50(8): 817-824.

Sharma, O.P., Bhosle, B.B., Kamble, K.R., Bhede, B.V. and Seeras, N.R. 2011. Management of pigeonpea pod borers with special reference to pod fly (Melanagromyza obtusa). Indian
Journal of Agricultural Sciences. 81(6): 539-543.

Singh, H.K. and Singh, H.N. 1990. Screening of certain pigeonpea cultivars own at kharif and rabi crops against tur pod bug, Clavigralla gibbosa and pod fly, Melanagromyza obtusa. Indian Journal of Entomology. 52: 320-327.

Singh, N., R.K. Tyagi and C. Pandey. 2013. Genetic Resources of Pigeonpea (Cajanus cajan): Conservation for Use. National Bureau of Plant Genetic Resources, New Delhi, India. pp. 49.

\section{How to cite this article:}

Badabagni Chiranjeevi, Neetin R. Patange and Prashant G. Chandile. 2018. Evaluation of Some Pigeonpea Genotypes for Their Response against Pod Fly, Melanagromyza obtusa (Malloch) Infestation. Int.J.Curr.Microbiol.App.Sci. 7(03): 1517-1526. doi: https://doi.org/10.20546/ijcmas.2018.703.181 